\title{
Asymptotic conditions for electromagnetic form factors of hadrons represented by VMD model
}

\author{
Cyril Adamuščin, Anna-Zuzana Dubničková \\ Dept. of Theoretical Physics, Comenius University, Mlynská dolina, 84248 Bratislava, \\ Slovak Republic \\ Stanislav Dubnička, Roman Pekárik \\ Institute of Physics, Slovak Academy of Sciences, Dúbravská cesta 9, 84228 Bratislava, \\ Slovak Republic. \\ and \\ Peter Weisenpacher \\ Institute of Informatics, Slovak Academy of Sciences, Dúbravská cesta 9, 84237 Bratislava, \\ Slovak Republic.
}

\begin{abstract}
A system of linear homogeneous algebraic equations for coupling constant ratios of vector-mesons to hadrons is derived by imposing an assumed asymptotic behaviour upon the VMD pole parametrization of an hadron electromagnetic form factor. A similar system of equations with a simpler structure of coefficients, taken as even powers of vector-meson masses, is derived by means of integral superconvergent sum rules for the imaginary part of the considered form factor using its appropriate $\delta$-function approximation. Although both systems have been derived starting from different properties of the electromagnetic form factor and they look each in its own way, it is shown explicitly that they are fully equivalent.
\end{abstract}

\section{Introduction}

In a construction of the unitary and analytic model [1-6] of the electromagnetic (EM) structure of an arbitrary hadron, the vector-meson-dominant (VMD) pole parametrization of EM form factors (FF's)

$$
F_{h}(t)=\sum_{v=1}^{n} \frac{m_{v}^{2}}{m_{v}^{2}-t}\left(f_{v h h} / f_{v}\right)
$$


is the starting point. The FF in this form is a pure real function in the whole physical region $-\infty<t<+\infty$, where $t=-Q^{2}$ is the squared momentum transfer of the virtual photon, $m_{v}$ are the masses of vector mesons and $f_{v h h}$ and $f_{v}$ are the coupling constants of vector-meson to hadron and vector-meson-photon transition, respectively. The expression (1) provides the basis for a consistent unification of all other FF properties, like asymptotics, analytic properties, unitarity, normalization etc, into an advanced unitary and analytic model.

Based on the so-called quark counting rules (QCR), the asymptotic behaviour of EM FF of a hadron is directly related $[7,8]$ to the number of constituent quarks $n_{q}$ by the expression

$$
F_{h}(t)_{|t| \rightarrow \infty} \sim t^{1-n_{q}}
$$

From Eq.(2) one can see immediately that for hadrons with $n_{q}>2$ the asymptotic behaviour of their EM FF's is different from the asymptotic behaviour of (1) and so, in the construction of an unitary and analytic model it has to be suitably adopted.

First, one transforms the VMD parametrization (1) into common denominator. As a result a rational function with a polynomial of $(n-1)$ degree in the numerator is obtained. Then a required asymptotic behaviour $\sim t^{-m}(m \leq n)$ of the considered FF is achieved by setting $(m-1)$ coefficients of the highest powers of the variable $t$ in the numerator to be zero.

In this manner the first system of $(m-1)$ linear homogeneous algebraic equations for coupling constant ratios with coefficients to be rather complicated sums of products of vectormeson masses squared is formulated.

On the other hand, let us assume that FF is analytic in the whole complex plane of its variable besides the cut on the positive real axis from $t_{0}$ to $\infty$ and possesses the asymptotic behaviour $\sim 1 / t^{m}$. Then one can apply the Cauchy theorem to the FF with an integration path being a circle of the radius $R \rightarrow \infty$, but avoiding the cut on the positive real axis. Because the integral along the circle with the infinity radius is zero, only integrals on the upper and lower boundary of the cut will contribute. By using the reality condition of FF they lead to the integral superconvergent sum rule for the imaginary part of FF.

The same procedure can be applied to the functions $t F_{h}(t), t^{2} F_{h}(t), \cdots, t^{m-2} F_{h}(t)$ which lead to another $(m-2)$ superconvergent sum rules for $t \operatorname{Im} F_{h}(t), t^{2} I m F_{h}(t), \cdots, t^{m-2} \operatorname{Im} F_{h}(t)$, respectively. Then approximating FF imaginary part by $\delta$ - function appropriately one ob- 
tains another system of $(m-1)$ linear algebraic equations for coupling constant ratios with coefficients that are simply even powers of the corresponding vector-meson masses.

In this paper we demonstrate that both systems of $(m-1)$ equations are equivalent.

The paper is organized as follows. In the next section we derive two systems of $(m-1)$ linear algebraic equations starting from different properties of electromagnetic FF of a hadron. Section 3 is devoted to an explicit proof of their equivalence. In the last section we present conclusions and discussion.

\section{Algebraic equations for coupling constant ratios}

Generally, let us assume that the FF in (11) is saturated by $n$ different vector meson pole terms and it has the asymptotic behaviour

$$
F_{h_{|t| \rightarrow \infty}} \sim t^{-m}
$$

where $m \leq n$.

Transforming the VMD pole representation (11) into a common denominator one obtains $\mathrm{FF}$ in the form of a rational function with a polynomial of $(m-1)$ degree

$$
P_{n-1}(t)=A_{0}+A_{1} \cdot t+A_{2} \cdot t^{2}+\cdots+A_{n-1} \cdot t^{n-1}
$$

in the numerator, where

$$
\begin{aligned}
A_{n-1} & =(-1)^{n-1} \sum_{j=1}^{n} m_{j}^{2} a_{j} \\
A_{n-2} & =(-1)^{n-2} \sum_{\substack{i=1 \\
i \neq j}}^{n} m_{i}^{2} \sum_{j=1}^{n} m_{j}^{2} a_{j} \\
A_{n-3} & =(-1)^{n-3} \sum_{\substack{i_{1}, i_{2}=1 \\
i_{1}<i_{2}, i_{r} \neq j}}^{n} m_{i_{1}}^{2} m_{i_{2}}^{2} \sum_{j=1}^{n} m_{j}^{2} a_{j} \\
A_{n-4} & =(-1)^{n-4} \sum_{\substack{i_{1}, i_{2}, i_{3}=1 \\
i_{1}<i_{2}<i_{3}, i_{r} \neq j}}^{n} m_{i_{1}}^{2} m_{i_{2}}^{2} m_{i_{3}}^{2} \sum_{j=1}^{n} m_{j}^{2} a_{j}
\end{aligned}
$$




$$
\begin{aligned}
A_{n-(m-1)}= & (-1)^{n-m+1} \sum_{\substack{i_{1}, i_{2}, \cdots i_{m-2}=1 \\
i_{1}<i_{2} \cdots i_{m-2}, i_{r} \neq j}}^{n} m_{i_{1}}^{2} m_{i_{2}}^{2} \cdots m_{i_{m-2}}^{2} \sum_{j=1}^{n} m_{j}^{2} a_{j} \\
A_{n-m}= & (-1)^{n-m} \sum_{\substack{i_{1}, i_{2}, \cdots i_{m-1}=1 \\
i_{1}<i_{2} \cdots<i_{m-1}, i_{r} \neq j}}^{n} m_{i_{1}}^{2} m_{i_{2}}^{2} \cdots m_{i_{m-2}}^{2} m_{i_{m-1}}^{2} \sum_{j=1}^{n} m_{j}^{2} a_{j} \\
A_{2}= & (-1)^{2} \sum_{\substack{i_{1}, i_{2}, \cdots i_{n-3}=1 \\
i_{1}<i_{2} \cdots<i_{n-3}, i_{r} \neq j}}^{n} m_{i_{1}}^{2} m_{i_{2}}^{2} \cdots m_{i_{n-3}}^{2} \sum_{j=1}^{n} m_{j}^{2} a_{j} \\
A_{1}= & (-1) \sum_{\substack{i_{1}, i_{2}, \cdots i_{n-2}=1 \\
i_{1}<i_{2} \cdots<i_{n-2}, i_{r} \neq j}}^{n} m_{i_{1}}^{2} m_{i_{2}}^{2} \cdots m_{i_{n-3}}^{2} m_{i_{n-2} 2}^{2} \sum_{j=1}^{n} m_{j}^{2} a_{j} \\
A_{0}= & \sum_{\substack{i_{1}, i_{2}, \cdots i_{n-1}=1 \\
i_{1}<i_{2} \cdots i_{n-1}, i_{r} \neq j}}^{n} m_{i_{1}}^{2} m_{i_{2}}^{2} \cdots m_{i_{n-2}}^{2} m_{i_{n-1}}^{2} \sum_{j=1}^{n} m_{j}^{2} a_{j}
\end{aligned}
$$

and $a_{j}=\left(f_{j h h} / f_{j}\right)$.

In order to achieve the assumed asymptotic behaviour (3) one requires in (4) the first $(m-1)$ coefficients from the highest powers of $t$ to be zero and as a result the following first system of linear homogeneous algebraic equations for the coupling constant ratios is obtained

$$
\begin{gathered}
\sum_{j=1}^{n} m_{j}^{2} a_{j}=0 \\
\sum_{\substack{i=1 \\
i \neq j}}^{n} m_{i}^{2} \sum_{j=1}^{n} m_{j}^{2} a_{j}=0 \\
\sum_{\substack{i_{1}, i_{2}=1 \\
i_{1}<i_{2}, i_{F} \neq j}}^{n} m_{i_{1}}^{2} m_{i_{2}}^{2} \sum_{j=1}^{n} m_{j}^{2} a_{j}=0 \\
\sum_{\substack{i_{1}, i_{2}, i_{3}=1 \\
i_{1}<i_{2}<i_{3}, i_{r} \neq j}}^{n} m_{i_{1}}^{2} m_{i_{2}}^{2} m_{i_{3}}^{2} \sum_{j=1}^{n} m_{j}^{2} a_{j}=0 \\
\sum_{\substack{n \\
i_{1}, i_{2}, \cdots i_{m-2}=1 \\
i_{1}<i_{2} \cdots<i_{m-2}, i_{r} \neq j}}^{n} m_{i_{1}}^{2} m_{i_{2}}^{2} \cdots m_{i_{m-2}}^{2} \sum_{j=1}^{n} m_{j}^{2} a_{j}=0 .
\end{gathered}
$$

As one can see from (6) with increased $m$ the coefficients become sums of more and more complicated products of squared vector-meson masses. 
For a derivation of the second system we employ the assumed analytic properties of EM FF's of hadrons, consisting of infinite number of branch points on the positive real axis, i.e. cuts. The first cut extends from the lowest branch point $t_{0}$ to $+\infty$. Then one can apply the Cauchy theorem to $\mathrm{FF}$ in $t$ - plane

$$
\frac{1}{2 \pi i} \oint F_{h}(t) d t=0
$$

where the closed integration path consists of the circle $C_{R}$ of the radius $R \rightarrow \infty$ and the path avoiding the cut on the positive real axis. As a result (7) can be rewritten into a sum of the following four integrals

$$
\frac{1}{2 \pi i}\left\{\int_{C_{R}} F_{h}(t) d t+\int_{+\infty}^{t_{0}} F_{h}(t-i \epsilon) d t+\int_{C_{r} / 2} F_{h}(t) d t+\int_{t_{0}}^{+\infty} F_{h}(t+i \epsilon) d t\right\}=0
$$

where $\epsilon \ll 1$ and $C_{r} / 2$ is the half-circle joining the upper boundary of the cut with the lower-boundary of the cut around the lowest branch point $t_{0}$. The contribution of the first integral in (8) is zero as $F_{h}(t)$ for $R \rightarrow \infty$ is vanishing. One can prove also that the third integral in (8) for $r \rightarrow 0$ is zero. As a result one gets

$$
\frac{1}{2 \pi i} \int_{t_{0}}^{\infty}\left[F_{h}(t+i \epsilon)-F_{h}(t-i \epsilon)\right] d t=0 .
$$

Then, taking into account the reality condition of FF

$$
F_{h}^{*}(t)=F_{h}\left(t^{*}\right)
$$

following from the general Schwarz reflection principle in the theory of analytic functions, one arrives at the integral superconvergent sum rule

$$
\frac{1}{\pi} \int_{t_{0}}^{\infty} \operatorname{Im} F_{h}(t) d t=0
$$

for the imaginary part of the FF under consideration.

Repeating the same procedure for the functions $t F_{h}(t), t^{2} F_{h}(t), \cdots, t^{m-2} F_{h}(t)$ which possess the same analytic properties in the complex $t$-plane as $F_{h}(t)$, one gets another $(m-2)$ superconvergent sum rules

$$
\begin{aligned}
\frac{1}{\pi} \int_{t_{0}}^{\infty} t \cdot I m F_{h}(t) d t & =0 \\
\frac{1}{\pi} \int_{t_{0}}^{\infty} t^{2} \cdot I m F_{h}(t) d t & =0
\end{aligned}
$$




$$
\frac{1}{\pi} \int_{t_{0}}^{\infty} t^{m-2} \cdot \operatorname{ImF}_{h}(t) d t=0 .
$$

Now, approximating the FF imaginary part by $\delta$ - function in the following form

$$
\operatorname{Im} F(t)=\pi \sum_{i}^{n} a_{i} \delta\left(t-m_{i}^{2}\right) m_{i}^{2}
$$

and substituting it into (11) and (12) one obtains the second system of $(m-1)$ linear homogeneous algebraic equations for coupling constant ratios $a_{i}=\left(f_{i h h} / f_{i}\right)$

$$
\begin{gathered}
\sum_{i=1}^{n} m_{i}^{2} a_{i}=0 \\
\sum_{i=1}^{n} m_{i}^{4} a_{i}=0 \\
\sum_{i=1}^{n} m_{i}^{6} a_{i}=0 \\
\ldots \ldots \ldots \\
\sum_{i=1}^{n} m_{i}^{2(m-2)} a_{i}=0 \\
\sum_{i=1}^{n} m_{i}^{2(m-1)} a_{i}=0,
\end{gathered}
$$

where coefficients are simply even powers of the vector-meson masses.

In the next section we demonstrate explicitly that both systems of the algebraic equations, (6) and (14), are equivalent.

\section{Equivalence of systems of algebraic equations for coupling constants ratios}

In this section we show step by step that the systems of linear algebraic equations (14) and (6), despite the fact that they have been derived starting from different properties of the EM $\mathrm{FF}$, and thus they appear to be different, are equivalent. As a consequence, in a constructing the unitary and analytic model of the EM structure of any hadron compound of more than two quarks one can employ instead of equations (6) the simpler set given by (14). 
We start with the equations (6). From a direct comparison of systems (6) and (14) one can see immediately the identity of the first equations in them.

The second equation in (6) can be written explicitly as follows $\left(m_{2}^{2}+m_{3}^{2}+\cdots+m_{n}^{2}\right) m_{1}^{2} a_{1}+\left(m_{1}^{2}+m_{3}^{2}+\cdots+m_{n}^{2}\right) m_{2}^{2} a_{2}+\cdots+\left(m_{1}^{2}+m_{2}^{2}+\cdots+m_{n-1}^{2}\right) m_{n}^{2} a_{n}=0$.

Adding and substracting $m_{1}^{4} a_{1}$ to the first term of the sum, $m_{2}^{4} a_{2}$ to the second term of the sum $\cdots$ etc. and finally $m_{n}^{4} a_{n}$ to the last term of the sum, the equation (15) can be modified into the form

$$
\sum_{i=1}^{n} m_{i}^{2} \sum_{j=1}^{n} m_{j}^{2} a_{j}-\sum_{j=1}^{n} m_{j}^{4} a_{j}=0,
$$

from where one can see immediately that the second equation in (14) is fulfilled

$$
\sum_{j=1}^{n} m_{j}^{4} a_{j}=0
$$

as $\sum_{j=1}^{n} m_{j}^{2} a_{j}=0$ is just the first equation in (6) and (14) as well.

The third equation in (6) can be written explicitly as follows

$$
\begin{aligned}
& \left(m_{2}^{2} m_{3}^{2}+m_{2}^{2} m_{4}^{2}+\cdots+m_{2}^{2} m_{n}^{2}+m_{3}^{2} m_{4}^{2}+m_{3}^{2} m_{5}^{2}+\cdots+m_{3}^{2} m_{n}^{2}+\cdots+m_{n-1}^{2} m_{n}^{2}\right) m_{1}^{2} a_{1}+ \\
+ & \left(m_{1}^{2} m_{3}^{2}+m_{1}^{2} m_{4}^{2}+\cdots+m_{1}^{2} m_{n}^{2}+m_{3}^{2} m_{4}^{2}+m_{3}^{2} m_{5}^{2}+\cdots+m_{3}^{2} m_{n}^{2}+\cdots+m_{n-1}^{2} m_{n}^{2}\right) m_{2}^{2} a_{2}+ \\
+ & \left(m_{1}^{2} m_{2}^{2}+m_{1}^{2} m_{4}^{2}+\cdots+m_{1}^{2} m_{n}^{2}+m_{2}^{2} m_{4}^{2}+m_{2}^{2} m_{5}^{2}+\cdots+m_{2}^{2} m_{n}^{2}+\cdots+m_{n-1}^{2} m_{n}^{2}\right) m_{3}^{2} a_{3}+ \\
& \cdots \cdots \\
& \cdots \\
+ & \left(m_{1}^{2} m_{2}^{2}+m_{1}^{2} m_{3}^{2}+\cdots+m_{1}^{2} m_{n-2}^{2}+m_{1}^{2} m_{n}^{2}+m_{2}^{2} m_{3}^{2}+\cdots+m_{2}^{2} m_{n-2}^{2}+m_{2}^{2} m_{n}^{2} \cdots+\right. \\
+ & \left.m_{n-2}^{2} m_{n}^{2}\right) m_{n-1}^{2} a_{n-1}+ \\
+ & \left(m_{1}^{2} m_{2}^{2}+m_{1}^{2} m_{3}^{2}+\cdots+m_{1}^{2} m_{n-2}^{2}+m_{1}^{2} m_{n-1}^{2}+m_{2}^{2} m_{3}^{2}+\cdots+m_{2}^{2} m_{n-2}^{2}+m_{2}^{2} m_{n-1}^{2}+\right. \\
+ & \left.\cdots+m_{n-2}^{2} m_{n-1}^{2}\right) m_{n}^{2} a_{n}=0 .
\end{aligned}
$$

Now adding and substracting all missing terms in (18) from $\sum_{\substack{i_{1}, i_{2}=1 \\ i_{1}<i_{2}}}^{n} m_{i_{1}}^{2} m_{i_{2}}^{2} \sum_{j=1}^{n} m_{j}^{2} a_{j}$ which in the substraction form can be written explicitly as follows

$$
\begin{aligned}
& -\left(m_{2}^{2}+m_{3}^{2}+m_{4}^{2}+\cdots m_{n}^{2}\right) m_{1}^{4} a_{1}- \\
& -\left(m_{1}^{2}+m_{3}^{2}+m_{4}^{2}+\cdots m_{n}^{2}\right) m_{2}^{4} a_{2}- \\
& -\left(m_{1}^{2}+m_{2}^{2}+m_{4}^{2}+\cdots m_{n}^{2}\right) m_{3}^{4} a_{3}-
\end{aligned}
$$




$$
\begin{aligned}
& -\left(m_{1}^{2}+m_{2}^{2}+\cdots+m_{n-2}^{2}+m_{n}^{2}\right) m_{n-1}^{4} a_{n-1}- \\
& -\left(m_{1}^{2}+m_{2}^{2}+\cdots+m_{n-2}^{2}+m_{n-1}^{2}\right) m_{n}^{4} a_{n}
\end{aligned}
$$

and again substracting and adding $m_{1}^{6} a_{1}$ in the first line of (19), $m_{2}^{6} a_{2}$ in the second line of (19) ...etc., and finally $m_{n}^{6} a_{n}$ in the last line of (19), one can rewrite (18) into the form

$$
\sum_{\substack{i_{1}, i_{2}=1 \\ i_{1}<i_{2}}}^{n} m_{i_{1}}^{2} m_{i_{2}}^{2} \sum_{j=1}^{n} m_{j}^{2} a_{j}-\sum_{i=1}^{n} m_{i}^{2} \sum_{j=1}^{n} m_{j}^{4} a_{j}+\sum_{j=1}^{n} m_{j}^{6} a_{j}=0 .
$$

From this expression, taking into account the first two equations in (14), the third equation of (14)

$$
\sum_{j}^{n} m_{j}^{6} a_{j}=0
$$

follows.

The fourth equation in (6) takes the following explicit form

$$
\begin{aligned}
& \left(m_{2}^{2} m_{3}^{2} m_{4}^{2}+\cdots+m_{2}^{2} m_{3}^{2} m_{n}^{2}+m_{2}^{2} m_{4}^{2} m_{5}^{2}+\cdots+m_{2}^{2} m_{4}^{2} m_{n}^{2}+\cdots+\right. \\
+ & \left.m_{n-2}^{2} m_{n-1}^{2} m_{n}^{2}\right) m_{1}^{2} a_{1}+ \\
+ & \left(m_{1}^{2} m_{3}^{2} m_{4}^{2}+\cdots+m_{1}^{2} m_{3}^{2} m_{n}^{2}+m_{1}^{2} m_{4}^{2} m_{5}^{2}+\cdots+m_{1}^{2} m_{4}^{2} m_{n}^{2}+\cdots+\right. \\
+ & \left.m_{n-2}^{2} m_{n-1}^{2} m_{n}^{2}\right) m_{2}^{2} a_{2}+ \\
+ & \left(m_{1}^{2} m_{2}^{2} m_{4}^{2}+\cdots+m_{1}^{2} m_{2}^{2} m_{n}^{2}+m_{1}^{2} m_{4}^{2} m_{5}^{2}+\cdots+m_{1}^{2} m_{4}^{2} m_{n}^{2}+\cdots+\right. \\
+ & \left.m_{n-2}^{2} m_{n-1}^{2} m_{n}^{2}\right) m_{3}^{2} a_{3}+ \\
& \quad \cdots \cdots+ \\
+ & \left(m_{1}^{2} m_{2}^{2} m_{3}^{2}+\cdots+m_{1}^{2} m_{2}^{2} m_{n}^{2}+m_{1}^{2} m_{3}^{2} m_{4}^{2}+\cdots+m_{1}^{2} m_{3}^{2} m_{n}^{2}+\cdots+\right. \\
+ & \left.m_{n-3}^{2} m_{n-2}^{2} m_{n}^{2}\right) m_{n-1}^{2} a_{n-1}+ \\
+ & \left(m_{1}^{2} m_{2}^{2} m_{3}^{2}+\cdots+m_{1}^{2} m_{2}^{2} m_{n-1}^{2}+m_{1}^{2} m_{3}^{2} m_{4}^{2}+\cdots+m_{1}^{2} m_{3}^{2} m_{n-1}^{2}+\cdots+\right. \\
+ & \left.m_{n-3}^{2} m_{n-2}^{2} m_{n-1}^{2}\right) m_{2}^{n} a_{n}=0
\end{aligned}
$$


First, adding and substracting all missing terms in (22) from

$$
\sum_{\substack{i_{1}, i_{2}, i_{3}=1 \\ i_{1}<i_{2}<i_{3}}}^{n} m_{i_{1}}^{2} m_{i_{2}}^{2} m_{i_{3}}^{2} \sum_{j=1}^{n} m_{j}^{2} a_{j},
$$

the equation (22) takes the form

$$
\begin{array}{r}
\sum_{\substack{i_{1}, i_{2}, i_{3}=1 \\
i_{1}<i_{2}<i_{3}}}^{n} m_{i_{1}}^{2} m_{i_{2}}^{2} m_{i_{3}}^{2} \sum_{j=1}^{n} m_{j}^{2} a_{j}- \\
-\quad \sum_{\substack{i_{1}, i_{2}=1 \\
i_{1}<i_{2}, i_{r} \neq j}}^{n} m_{i_{1}}^{2} m_{i_{2}}^{2} \sum_{j=1}^{n} m_{j}^{4} a_{j}=0 .
\end{array}
$$

Second, substracting and adding of all missing terms in (23) from $\sum_{\substack{i_{1}, i_{2}=1 \\ i_{1}<i_{2}}}^{n} m_{i_{1}}^{2} m_{i_{2}}^{2} \sum_{j=1}^{n} m_{j}^{4} a_{j}$ one gets the equation

$$
\begin{gathered}
\sum_{\substack{i_{1}, i_{2}, i_{3}=1 \\
i_{1}<i_{2}<i_{3}}}^{n} m_{i_{1}}^{2} m_{i_{2}}^{2} m_{i_{3}}^{2} \sum_{j=1}^{n} m_{j}^{2} a_{j}- \\
-\sum_{\substack{i_{1}, i_{2}=1 \\
i_{1}<i_{2}}}^{n} m_{i_{1}}^{2} m_{i_{2}}^{2} \sum_{j=1}^{n} m_{j}^{4} a_{j}+\sum_{\substack{i=1 \\
i \neq j}}^{n} m_{i}^{2} \sum_{j=1}^{n} m_{j}^{6} a_{j}=0 .
\end{gathered}
$$

Finally, additions and substractions of all missing terms in (24) from $\sum_{i=1}^{n} m_{i}^{2} \sum_{j=1}^{n} m_{j}^{6} a_{j}$ lead to the definitive form of the fourth equation in (6)

$$
\begin{aligned}
& \sum_{\substack{i_{1}, i_{2}, i_{3}=1 \\
i_{1}<i_{2}<i_{3}}}^{n} m_{i_{1}}^{2} m_{i_{2}}^{2} m_{i_{3}}^{2} \sum_{j=1}^{n} m_{j}^{2} a_{j}- \\
&-\sum_{\substack{i_{1}, i_{2}=1 \\
i_{1}<i_{2}}}^{n} m_{i_{1}}^{2} m_{i_{2}}^{2} \sum_{j=1}^{n} m_{j}^{4} a_{j}+\sum_{i=1}^{n} m_{i}^{2} \sum_{j=1}^{n} m_{j}^{6} a_{j}-\sum_{j=1}^{n} m_{j}^{8} a_{j}=0 .
\end{aligned}
$$

From here, taking into account the first three equations in (14), the fourth equation in (14)

$$
\sum_{j=1}^{n} m_{j}^{8} a_{j}=0
$$

follows.

It is now easy to give a straightforward generalization of the above procedures:

i) the q-th equation in (6) can be decomposed into q-terms (see (16), (20) and (25)) consisting of the product of two parts, where the first part is just the sum of decreasing 
numbers of products of different vector-meson masses squared, starting from (q-1) coefficients and ending with the constant 1 . The second term takes the form $\sum_{j=1}^{n} m_{j}^{\alpha} a_{j}$ with increasing even power $\alpha$ starting from $\alpha=2$ up to $2 \mathrm{q}$.

ii) there is an alternating sign in front of every term in that decomposition, while the first term is always positive.

Now, in order to carry out a general proof of the equivalence of the two systems of algebraic equations under consideration, let us assume an equivalence of $(m-2)$ equations in (6) and (14). Then, taking into account a generalization of our procedure defined by rules i) and $i i)$ above, one can decompose the $(m-1)$-equation in (6) into the following form

$$
\begin{aligned}
& \sum_{\substack{i_{1}, i_{2}, i_{3}, \cdots, i_{m-2}=1 \\
i_{1}<i_{2}<i_{3}<\cdots<i_{m-2}}}^{n} m_{i_{1}}^{2} m_{i_{2}}^{2} \cdots m_{i_{m-2}}^{2} \sum_{j}^{n} m_{j}^{2} a_{j}- \\
& -\sum_{\substack{i_{1}, i_{2}, i_{3}, \cdots, i_{m-3}=1 \\
i_{1}<i_{2}<i_{3}<\cdots<i_{m-3}}}^{n} m_{i_{1}}^{2} m_{i_{2}}^{2} \cdots m_{i_{m-3}}^{2} \sum_{j=1}^{n} m_{j}^{4} a_{j}+ \\
& +\quad \sum_{\substack{i_{1}, i_{2}, i_{3}, \cdots, i_{m-4}=1 \\
i_{1}<i_{2}<i_{3}<\cdots<i_{m-4}}}^{n} m_{i_{1}}^{2} m_{i_{2}}^{2} \cdots m_{i_{m-4}}^{2} \sum_{j}^{n} m_{j}^{6} a_{j}+\cdots+ \\
& +\quad(-1)^{m-3} \sum_{i=1}^{n} m_{i}^{2} \sum_{j=1}^{n} m_{j}^{2(m-2)} a_{j}+(-1)^{m-2} \sum_{j=1}^{n} m_{j}^{2(m-1)} a_{j}=0,
\end{aligned}
$$

from where one can see immediately that the $(m-1)$ equation in (14) is satisfied

$$
\sum_{j=1}^{n} m_{j}^{2(m-1)} a_{j}=0
$$

as $\sum_{j=1}^{n} m_{j}^{2} a_{j}=0, \sum_{j=1}^{n} m_{j}^{4} a_{j}=0, \cdots, \sum_{j=1}^{n} m_{j}^{2(m-2)} a_{j}=0$ are just the first $(m-2)$ equations in (14) assumed to be valid.

At the end we would like to draw an attention to the proof of the equivalence of the systems of algebraic equations (6) and (14) from the other point of view.

If the sums $\sum_{j=1}^{n} m_{j}^{2} a_{j}, \sum_{j=1}^{n} m_{j}^{4} a_{j}, \sum_{j=1}^{n} m_{j}^{6} a_{j}, \cdots, \sum_{j=1}^{n} m_{j}^{2(m-3)} a_{j}, \sum_{j=1}^{n} m_{j}^{2(m-2)} a_{j}$, $\sum_{j=1}^{n} m_{j}^{2(m-1)} a_{j}$ are considered to be independent variables, then the first equation in (6) together with the modified forms $(16),(20),(25), \ldots, 27)$ form a system of $(m-1)$ homogeneous algebraic equations for these variables and the equations (14) are just its trivial solutions. 


\section{Conclusions and discussion}

Starting from different properties of the EM FF of hadron we have derived two apparently distinct systems of linear homogeneous algebraic equations for the coupling constant ratios of vector-mesons to the hadron under consideration.

For a derivation of the first system of equations we have assumed that EM FF of hadron is well approximated by a finite number of vector-meson exchange tree Feynman diagrams leading to the VMD pole parametrization of $\mathrm{FF}$. A subsequent requirement of a true asymptotics of EM FF gives the system of linear homogeneous algebraic equations for coupling constant ratios with coefficients that are rather complicated sums of products of squared vector-meson masses.

For a derivation of the second system of equations analytic properties together with the asymptotic behaviour of EM FF have been utilized. The application of Cauchy theorem to $F_{h}(t), t F_{h}(t), t^{2} F_{h}(t), \cdots, t^{m-2} F_{h}(t)$ leads to $(m-1)$ integral superconvergent sum rules for $I m F_{h}(t), t I m F_{h}(t), t^{2} I m F_{h}(t), \cdots, t^{m-2} I m F_{h}(t)$. Then an appropriate approximation of $\mathrm{FF}$ imaginary part by $\delta$-function gives another system of linear homogeneous algebraic equations for coupling constant ratios with coefficients to be simply even powers of the vector-meson masses.

By using a sequence of algebraic manipulations it has been proved step by step that both systems of equations for coupling constant ratios are equivalent.

Finally a natural question arises about a practical application of such systems of equations for coupling constant ratios. The latter was already indicated to some extent in the introduction, but in relation to this issue some peculiarities have to be mentioned. The asymptotic behaviour of EM FF is given by a number $n_{q}$ of the constituent quarks in the hadron and so, the system of algebraic equations for coupling constant ratios can be derived only in the case if $n_{q}>2$. However, a necessary condition for the latter is saturation of the sum in (1) by a number of vector-meson resonances $n$ to be greater or equal to $n_{q}-1$.

If $n>n_{q}-1$, then the derived system of equations leads in a constructed unitary and analytic model of EM structure of the hadron to a remarkable reduction of a number of free coupling constant ratios. 
If $n=n_{q}-1$, then adding to $(n-1)$ algebraic equations the equation following from a normalization of $F_{h}(t)$ at $t=0$ that typically has a non-zero value, one obtains inhomogeneous system of $n$ linear algebraic equations with $n$ variables that can be non-trivially solved for. Then solutions are just numerical values of coupling constant ratios, which appear to be in very good approximation to the physical reality (for the case of nucleons see [9]).

The inhomogeneous system of $n \geq n_{q}-1$ linear algebraic equations is a very natural tool for a successful simultaneous incorporation of the normalization and the true asymptotics of $F_{h}(t)$ into the unitary and analytic model of EM structure of any hadron with $n_{q}>2$.

The work was in part supported in by Slovak Grant Agency for Sciences, Grant No. 2/5085/20 (S.D.) and Grant No. 1/7068/20 (A.Z.D)

\section{References}

[1] S. Dubnička, Nuovo Cim. A100, 1 (1988)

[2] S. Dubnička, A. Z. Dubničková, P. Striženec, Nuovo Cim. A106, 1253 (1993)

[3] S. Dubnička, A. Z. Dubničková, J. Kraskiewicz, R. Razcka, Z. Phys. C60, 153 (1995)

[4] S. Dubnička, $\pi \mathrm{N}$ Newsletter 11, 167 (1995)

[5] S. Dubnička, Acta Physica Polonica B27, 2525 (1996)

[6] S. Dubnička, A. Z. Dubničková, P. Striženec, hep-ph/0108053 and in Proceedings of the International Conference 'Hadron Structure 2000', Stará Lesná, 2000, edited by A.Z.Dubničková, S.Dubnička, P.Striženec (Comenius University, Bratislava 2001), p.204

[7] V. A. Matveev, R. M. Muradyan, A. N. Tavkhelidze, Lett. Nuovo Cim. 7, 719 (1973)

[8] S. J. Brodsky, G. R. Farrar, Phys. Rev. Lett. 31, 1153 (1973)

[9] S. Dubnička, A. Z. Dubničková, E. Krnáč, Phys. Lett B261, 127 (1991) 